\title{
A promising magnetic resonance stem cell tracer based on natural biomaterials in a biological system: manganese(II) chelated to melanin nanoparticles
}

This article was published in the following Dove Press journal:

International Journal of Nanomedicine

\author{
Shi-Jie Liu',2,* \\ Ling-Jie Wangl,* \\ Ying Qiao' \\ Hua Zhang' \\ Li-Ping $\mathrm{Li}^{\prime}$ \\ Jing-Hua Sun' \\ Sheng $\mathrm{He}^{1}$ \\ Wen $\mathrm{Xu}^{1,2}$ \\ Xi Yang ${ }^{\prime}$ \\ Wen-Wen Cai ${ }^{2}$ \\ Jian-Ding $\mathrm{Li}^{\prime}$ \\ Bin-Quan Wang ${ }^{3}$ \\ Rui-Ping Zhang ${ }^{2}$
}

'Medical Imaging Department, First Clinical Medical College of Shanxi Medical University, Taiyuan, Shanxi, China; ${ }^{2}$ Imaging Department, Affiliated Tumor Hospital of Shanxi Medical University, Taiyuan, Shanxi, China; ${ }^{3}$ Department of Otolaryngology, Head \& Neck Surgery, The First Hospital of Shanxi Medical University, Taiyuan, Shanxi, China

*These authors contributed equally to the paper
Correspondence: Rui-Ping Zhang Imaging Department, Affiliated Tumor Hospital of Shanxi Medical University, Number 3, Zhigongxin Road, Taiyuan, Shanxi, 03000I, China Email zrp_7I42@I63.com
Background: Melanin and manganese are both indispensable natural substances that play crucial roles in the human body. Melanin has been used as a multimodality imaging nanoplatform for biology science research because of its natural binding ability with metal ions (eg, ${ }^{64} \mathrm{Cu}^{2+}, \mathrm{Fe}^{3+}$, and $\mathrm{Gd}^{3+}$ ). Because of its effects on T1 signal enhancement, Mn-based nanoparticles have been used in magnetic resonance (MR) quantitative cell tracking in vivo. Stem cell tracking in vivo is an essential technology used to characterize engrafted stem cells, including cellular viability, biodistribution, differentiation capacity, and long-term fate.

Methods: In the present study, manganese(II) ions chelated to melanin nanoparticles [MNP$\mathrm{Mn}(\mathrm{II})]$ were synthesized. The characteristics, stem cell labeling efficiency, and cytotoxicity of the nanoparticles were evaluated. MR imaging of the labeled stem cells in vivo and in vitro were also further performed. In T1 relaxivity (r1), MNP-Mn(II) were significantly more abundant than Omniscan. Bone marrow-derived stem cells (BMSCs) can be labeled easily by coincubating with MNP-Mn(II), suggesting that MNP-Mn(II) had high biocompatibility.

Results: Cell Counting Kit- 8 assays revealed that MNP-Mn(II) had almost no cytotoxicity when used to label BMSCs, even with a very high concentration $(1,600 \mu \mathrm{g} / \mathrm{mL})$. BMSCs labeled with MNP-Mn(II) could generate a hyperintense T1 signal both in vitro and in vivo, and the hyperintense $\mathrm{T} 1$ signal in vivo persisted for at least 28 days.

Conclusion: Taken together, our results showed that MNP-Mn(II) possessed many excellent properties for potential quantitative stem cell tracking in vivo.

Keywords: melanin, manganese, magnetic resonance imaging, mesenchymal stem cells, cell tracer

\section{Introduction}

Stem cell transplantation has been used for the treatment of various diseases, ${ }^{1-5}$ owing to their advantageous properties, including multidifferentiation potential, self-renewal ability, the capacity to localize to the lesion area, and the capacity to act as a functional substitute for damaged cells. ${ }^{6}$ However, the characteristics of engrafted stem cells, including cellular viability, biodistribution, differentiation capacity, and long-term fate, have not yet been determined. ${ }^{6}$ Therefore, sequential visualization of engrafted stem cells in vivo is necessary to explore these mechanisms. ${ }^{7}$

Currently, magnetic resonance imaging (MRI) is commonly used for noninvasive cell tracking in vivo. Recently, various types of nanoparticles have been utilized for cell labeling and cellular MRI to study stem cell transplantation, including Gd-loaded nanoparticles, ${ }^{8,9}$ iron oxide nanoparticles, ${ }^{10}$ silica nanoparticles, ${ }^{11,12}$ quantum dots, ${ }^{13}$ and upconversion nanoparticles. ${ }^{14,15}$ However, most of these particles are not ideal 
MR cell tracers, owing to inefficient cellular labeling, biotoxicity, or low relaxivity. For example, Gd(III)-based agents may produce nephrotoxicity, ${ }^{16}$ and the dark signals produced by superparamagnetic iron oxide-based nanoparticles in T2-weighted images are difficult to distinguish from other tissues (such as the liver and spleen), physiological conditions (such as flow-void effects and air chambers), and diseases (such as hemorrhage and melanoma). ${ }^{17,18}$ Thus, many attempts to overcome such disadvantages have been made by searching for appropriate synthetic materials for nanoparticles to apply to cellular MRI.

Manganese is an essential element in humans, and is mainly distributed in the muscles, liver, kidneys, and brain in the form of manganese(II) ions. Manganese(II) can pass through calcium ion voltage-gated channels into the cell. ${ }^{19}$ Hence, manganese(II) possesses much better biocompatibility than other paramagnetic metal ions, ${ }^{20}$ particularly Gd(III) ions, which are widely applied as a clinical MR contrast agent.

Melanin is a ubiquitous pigment and an important chromophore in the human body. Melanin is produced by melanocytes, and is present in many human organs and tissues, including the skin, mucous membranes, retina, cerebral pia mater, and ovary. ${ }^{21,22}$ Melanin has many favorable properties, eg, good biocompatibility and metal ion chelation. Therefore, in recent years, increasing studies have explored the use of melanin in biological applications. ${ }^{22-24}$

Melanin nanoparticles have O-phenolic hydroxyl groups, which can easily chelate metal ions, such as ${ }^{64} \mathrm{Cu}^{2+}, \mathrm{Fe}^{3+}$, $\mathrm{Gd}^{3+}$, and $\mathrm{Mn}^{2+} \cdot{ }^{24}$ Manganese(II) ions chelated to melanin nanoparticles [MNP-Mn(II)] are a novel type of nanoparticles used for stem cell MR tracking. These nanoparticles exhibit an ultrasmall size, minimal cytotoxicity, high relaxivity, and efficient and long-term cell labeling capacity. In this study, we report the development and characterization of these nanoparticles for stem cell labeling and cellular MR imaging of engrafted stem cells. All components of these nanoparticles were endogenous substances derived from humans. Our findings provide important insights into the potential applications of MNP-Mn(II) as novel cell MRI tracers in vivo.

\section{Methods}

\section{Synthesis and characterization of MNP-Mn(II)}

Details of the synthesis and characterization of MNP-Mn(II) complexes are provided in the Supplementary materials.

\section{MRI of MNP-Mn(II) aqueous solution}

Samples of MNP-Mn(II) and Omniscan (an MRI contrast agent commonly used in the clinic) aqueous solutions were imaged using a clinical 3.0T instrument (Trivo; Siemens, Munich, Germany) with a wrist surface coil (diameter: $11 \mathrm{~cm}$ ). The samples of MNP-Mn(II) and Omniscan aqueous solutions were placed in polymerase chain reaction (PCR) plates at different $\mathrm{Mn}(\mathrm{II})$ and $\mathrm{Gd}(\mathrm{III})$ concentrations $(0.1,0.2$, $0.4,0.8$, and $1.0 \mathrm{mM}$ ), and pure water was used as a negative control. The concentrations of $\mathrm{Mn}$ (II) and Gd(III) in aqueous solution were measured by inductively coupled plasmaatomic emission spectrometry. T1-weighted imaging (T1WI) was performed twice using different repetition times (300 and $100 \mathrm{~ms}$ ) to measure the T1 value. Other T1WI parameters were as follows: spin-echo sequence, field of view (FOV): $90 \times 60 \mathrm{~mm}^{2}$; slice thickness: $1.5 \mathrm{~mm}$; spacing: $0.5 \mathrm{~mm}$; base matrix resolution: $256 \times 256$; and effective echo time: $20 \mathrm{~ms}$. The T1 relaxation efficient (r1) values of MNP-Mn(II) and Omniscan were obtained by calculating the ratio of $1 / \mathrm{T} 1$ [s-1] to the $\mathrm{Mn}(\mathrm{II})$ and $\mathrm{Gd}(\mathrm{III})$ concentrations (mM).

\section{Ethics statement}

All animal experiments was approved by the Institutional Animal Care and Use Committee of Shanxi Medical University (approval no 2016LL141) and complied with the US Guide for the Care and Use of Laboratory Animals, 8th Edition, 2011. ${ }^{25}$

\section{Bone marrow-derived stem cell (BMSC) labeling}

BMSCs were harvested by primary culture, as described in our previous study. ${ }^{26}$ Briefly, BMSCs were incubated in $25-\mathrm{cm}^{2}$ tissue culture flasks and, after reaching 70\%-80\% confluence, the medium was replaced with fresh complete culture medium containing MNP-Mn(II) at a concentration of $800 \mu \mathrm{g} / \mathrm{mL}$. The cells were subsequently cultured for $24 \mathrm{~h}$ at $37^{\circ} \mathrm{C}$ in a $5 \% \mathrm{CO}_{2}$ incubator. The medium was then discarded, and the cells were carefully rinsed five times with phosphate-buffered saline (PBS) to remove the unlabeled MNP-Mn(II), followed by trypsinization. The pooled cells were obtained by centrifugation $(1,000 \times g, 5 \mathrm{~min})$ and then resuspended in $1 \mathrm{~mL}$ serum-free culture medium for future analysis.

\section{Confocal fluorescence microscopy analysis}

MNP-Mn(II) (5 mg) and rhodamine-B (0.2 mg) were mixed in $5 \mathrm{~mL}$ water and stirred for $1 \mathrm{~h}$ at $40^{\circ} \mathrm{C}$ using a magnetic 
stirrer. The chelator MNP-Mn(II)-rhodamine-B was obtained though intermolecular crosslinking and electric attraction. The obtained MNP-Mn(II)-rhodamine-B aqueous solution was washed several times with ultrapure water in an ultrafiltration centrifuge tube (Amicon Ultra, $30 \mathrm{kDa}$; Millipore, Billerica, MA, USA) by centrifugation $(4,000-5,000 \times \mathrm{g}$, $15 \mathrm{~min}$ ) to remove unchelated rhodamine-B.

BMSCs were incubated with MNP-Mn(II)-rhodamine-B $(200 \mu \mathrm{g} / \mathrm{mL})$ for $4 \mathrm{~h}$ at $37^{\circ} \mathrm{C}$. The cells were then washed with PBS three times and fixed with $4 \%$ paraformaldehyde for $15 \mathrm{~min}$ at $4^{\circ} \mathrm{C}$. After drying, the cell nuclei were stained with 4',6-diamidino-2-phenylindole (DAPI). Finally, the fluorescence images were recorded and analyzed by confocal laser-scanning microscope (FV1000; Olympus, Tokyo, Japan).

\section{Transmission electron microscopy (TEM)}

TEM was used to visualize the intracellular distribution of MNP-Mn(II). For this, MNP-Mn(II)-labeled and unlabeled cells were resuspended and centrifuged at $1,500 \times g$ for $5 \mathrm{~min}$, yielding cell pellets. After the supernatants were removed, cell pellets were fixed at $4^{\circ} \mathrm{C}$ with $2 \%$ glutaraldehyde for $1 \mathrm{~h}$, and washed with PBS several times. The cells were then post-fixed with $1 \% \mathrm{OsO}_{4}$ for $1 \mathrm{~h}$, dehydrated in a graded series of ethanol solutions. The cells in ultrathin sections were obtained using an ultramicrotome (Leica EM UC-6; Leica Microsystems, Mannheim, Germany) and stained with uranyl acetate and lead citrate. Finally, the sections were visualized using a TEM (JEM-1011; JEOL).

\section{In vitro cytotoxicity assessment}

To assess the cytotoxicity of MNP-Mn(II), Cell Counting Kit-8 (CCK-8) assays were performed. Briefly, BMSCs were trypsinized and seeded on 96 -well plates $\left(5 \times 10^{3}\right.$ cells/well). Cells were cultured overnight, and the medium was replaced with fresh Dulbecco's Modified Eagle's Medium/F12 containing MNP-Mn(II) or MNPs at various concentrations $(0,25,50,100,200,400,800$, and $1,600 \mu \mathrm{g} / \mathrm{mL})$, followed by incubation for $48 \mathrm{~h}$ at $37^{\circ} \mathrm{C}$ in a $5 \% \mathrm{CO}_{2}$ incubator. Culture medium containing MNP-Mn(II) was then discarded, and the cells were subsequently washed three times with PBS. Fresh culture medium containing $10 \mu \mathrm{L}$ CCK-8 reagent was added into the wells and incubated for $4 \mathrm{~h}$ at $37^{\circ} \mathrm{C}$ in a $5 \%$ $\mathrm{CO}_{2}$ incubator.

Finally, the absorbance of each well was measured at $450 \mathrm{~nm}$ and recorded using a microplate reader (Infinite M1000; TECAN, Morrisville, NC, USA). All samples were assayed in triplicate.

\section{In vitro MRI}

After incubation with various concentrations of MNP-Mn(II) $(0,50,100,200,400$, and $800 \mu \mathrm{g} / \mathrm{mL})$ for $24 \mathrm{~h}$, cells were centrifuged and fixed with $4 \%$ paraformaldehyde for $20 \mathrm{~min}$ at $4{ }^{\circ} \mathrm{C}$. The cells were then resuspended and transferred to 96 -well PCR plates at a density of $1.5 \times 10^{6}$ cells/well. T1-weighted imaging of the cells was performed using a clinical 3.0T MRI instrument (Trivo; Siemens) with a wrist surface coil (diameter: $11 \mathrm{~cm}$ ). The detailed parameters were as follows: spin-echo sequence; FOV: $60 \times 90 \mathrm{~mm}$; matrix size: $180 \times 256$; slice thickness: $1.5 \mathrm{~mm}$; spacing: $0.5 \mathrm{~mm}$; TR: $500 \mathrm{~ms}$; and TE: $20 \mathrm{~ms}$.

\section{In vivo MRI of engrafted BMSCs}

A total of four male Sprague-Dawley rats (Animal Center of Shanxi Medical University, Taiyuan, China; SYXK20150001; 6 weeks of age, weighing 70-80 g each) were used in this study.

All rats were intraperitoneally administered $1 \%$ pentobarbital sodium $(40 \mathrm{mg} / \mathrm{kg})$ for anesthetization. Intramuscular injection of a $100-\mu \mathrm{L}$ suspension containing $2 \times 10^{6}$ BMSCs labeled with or without MNP-Mn(II) was then performed into the right hip. The injection positions were marked on the skin. MRI of the rats was carried out using a clinical 3.0T MRI instrument (Trivo; Siemens) with a wrist surface coil (diameter: $11 \mathrm{~cm}$ ) on day 1 before injection and on days $1,7,10,14,21$, and 28 after BMSC injection. The imaging parameters were the same as those of the above-mentioned in vitro MRI experiments.

The signal intensities (SIs) of the MNP-Mn(II)-labeled BMSC injection region and the adjacent soft tissue were measured, and the relative SIs were calculated to further evaluate the imaging capability of MNP-Mn(II)-labeled BMSCs in vivo. The area of the hyperintense signal generated by MNP-Mn(II)-labeled BMSCs was also measured at the same time.

\section{Results and discussion}

As shown in Figure S1, MNP-Mn(II) nanoparticles exhibited many excellent properties, such as good water solubility, high stability, and ultrasmall and uniform particle size. Nonetheless, an MR-positive cell tracer must have high longitudinal relaxivity. To evaluate the T1-weighted imaging capability of MNP-Mn(II), we investigated the T1-weighted image SIs of MNP-Mn(II) and Omniscan aqueous solutions with various concentrations. As shown in Figure 1A, the SI of the MNP-Mn(II) aqueous solution was significantly higher than that of the Omniscan aqueous solution at the same molar 

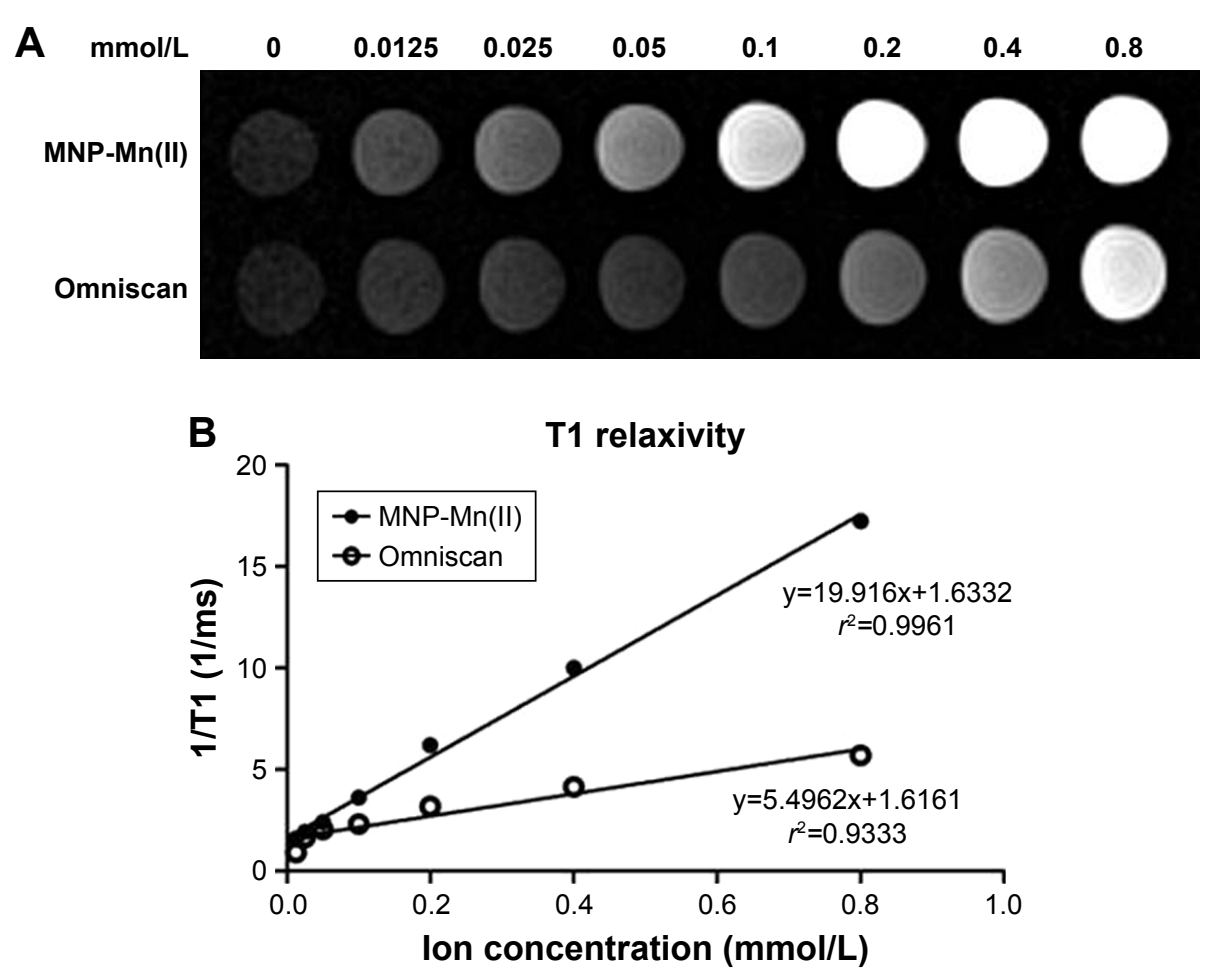

Figure I (A) TI-weighted MRI of MNP-Mn(II) and Omniscan aqueous solutions. (B) Plots of TI relaxation rates (I/TI) for MNP-Mn(II) and Omniscan aqueous suspensions based on metal ion (ie, $\mathrm{Mn}^{2+}, \mathrm{Gd}^{3+}$ ) concentrations (mM). The slope of each regression represents the relaxivity (ie, $\mathrm{rl}$ ).

Abbreviations: MNP-Mn(II), manganese (II) ions chelated to melanin nanoparticles; MRI, magnetic resonance imaging.

concentration. Owing to the intrinsic paramagnetic properties of the Mn(II) ion, MNP-Mn(II) exhibited excellent T1 relaxation efficacy. The $\mathrm{r} 1$ values, which represented the longitudinal relaxation of MNP-Mn(II) and Omniscan, were calculated from the slopes of the ion concentration-dependent relaxation 1/T1. As shown in Figure 1B, T1 relaxivity was significantly higher for MNP-Mn(II) (r1 = 19.916/mmol/s) than for Omniscan $(\mathrm{r} 1=5.4962 / \mathrm{mmol} / \mathrm{s})$ under the same testing conditions. These results further suggested that MNP$\mathrm{Mn}(\mathrm{II})$ had the potential to be applied to MR cell tracking in vivo.

Excellent cell tracers must also show effective cellular labeling and low cytotoxicity. ${ }^{27}$ Therefore, the cell labeling capability of MNP-Mn(II) was investigated using rhodamine staining and TEM, and cytotoxicity was evaluated with CCK-8 assays. Laser scanning microscope images (Figure 2) showed that many red fluorescent particles representing MNP-Mn(II)-rhodamine were present in the cytoplasm. TEM images (Figure 3A and B) also showed that many black particles representing MNP-Mn(II) aggregated in the cytoplasm and lysosome of BMSCs, and there were no black particles in the cytoplasm or endosomes of unlabeled BMSCs. Fontana-Masson staining was further performed to evaluate the labeling rate of cells (Figure S2). From these images, we found that almost all of the stem cells were successfully labeled with MNP-Mn(II) by
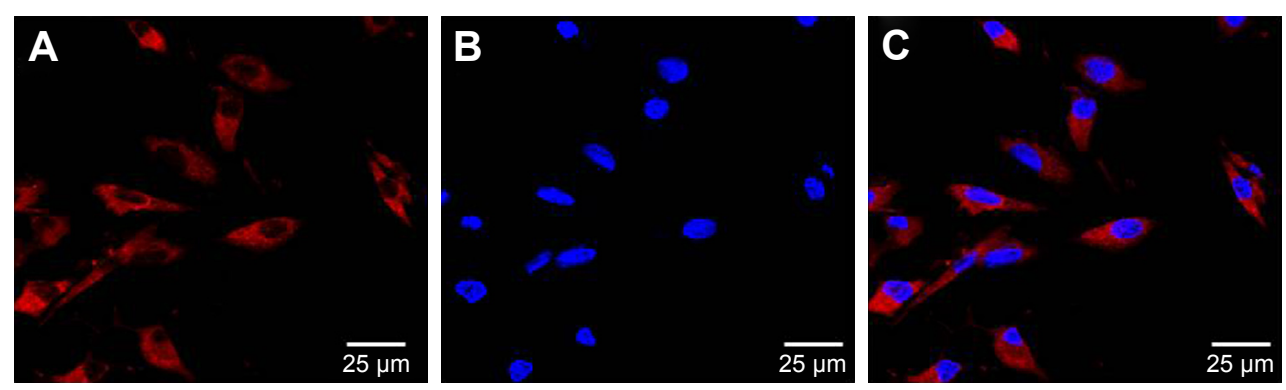

Figure 2 Confocal microscopy image of MNP-Mn(II)-rhodamine-B-labeled BMSCs. (A) Rhodamine (red) in the cytoplasm, (B) cell nuclei (stained by DAPI, blue), and (C) merged image; scale bars $=25 \mu \mathrm{m}$.

Abbreviations: BMSCs, bone marrow-derived stem cells; DAPI, 4',6-diamidino-2-phenylindole; MNP-Mn(II), manganese (II) ions chelated to melanin nanoparticles. 


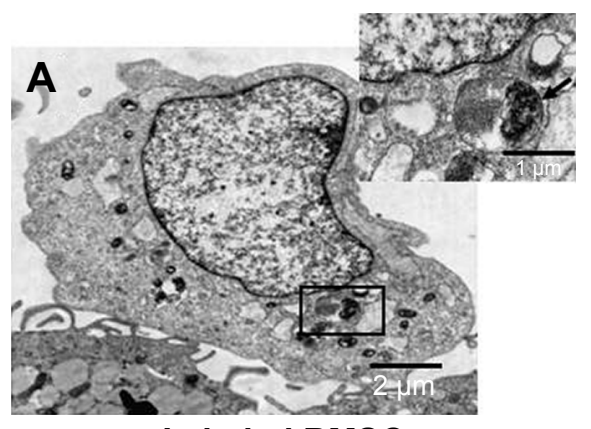

Labeled BMSCs

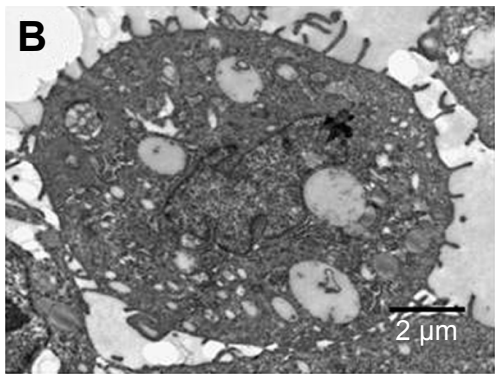

Unlabeled BMSCs

Figure 3 TEM images of MNP-Mn(II)-labeled BMSCs and unlabeled BMSCs; scale bars $=2 \mu \mathrm{m}$. (A) Black particles representing the internalized MNP-Mn(II) were observed in the cytoplasm and endosomes of labeled BMSCs (enlarged in the upper right corner). (B) No black particles were observed in endosomes of unlabeled BMSCs.

Abbreviations: BMSCs, bone marrow-derived stem cells; MNP-Mn(II), manganese (II) ions chelated to melanin nanoparticles; TEM, transmission electron microscopy.

co-incubation alone, without the need to use any transfection agents, demonstrating the excellent affinity of MNP-Mn(II) to the cells. As shown in Figure 4, BMSCs labeled with $1,600 \mu \mathrm{g} / \mathrm{mL}$ MNP-Mn(II) exhibited very high cell viability. These results demonstrated that MNP-Mn(II) could be easily taken up and internalized by BMSCs without the aid of transfection agents, and that these nanoparticles had almost no cytotoxicity.

Based on these properties of MNP-Mn(II), we further assessed the in vitro and in vivo MRI of BMSCs labeled with MNP-Mn(II). As shown in Figure 5, the cells were labeled with $0,100,200,400$, or $800 \mu \mathrm{g} / \mathrm{mL}$ MNP-Mn(II), and we found that cells labeled with $400 \mu \mathrm{g} / \mathrm{mL}$ exhibited the highest T1 SI at all labeling concentrations. However, the T1 SI at $800 \mu \mathrm{g} / \mathrm{mL}$ was lower than that at $400 \mu \mathrm{g} / \mathrm{mL}$, potentially because the high concentration of MNP-Mn(II)

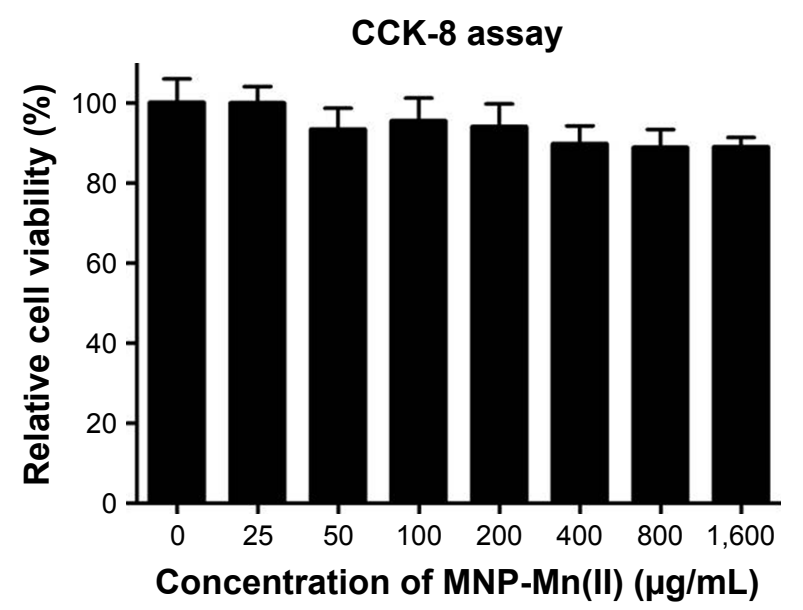

Figure 4 Cytotoxicity assessment of BMSCs labeled with MNP-Mn(II) at various concentrations $(0,25,50,100,200,400,800$, and $1,600 \mu \mathrm{g} / \mathrm{mL})$ was performed using CCK-8 assays. The relative cell viability was estimated by measuring the optical density of each well at $450 \mathrm{~nm}$.

Abbreviations: BMSCs, bone marrow-derived stem cells; CCK-8, Cell Counting Kit-8; MNP-Mn(II), manganese (II) ions chelated to melanin nanoparticles. in the cell may have shortened the transverse relaxation time. Thus, the peak of the signal could not be detected using the MR equipment, and the SI of the T1 images decreased. ${ }^{28}$ This phenomenon was also observed for other paramagnetic contrast agents. ${ }^{29}$

To further assess the capability of BMSCs labeled with MNP-Mn(II) for in vivo MRI, labeled BMSCs were intramuscularly injected into the right hips of rats. As shown in Figure 6A, a hyperintense T1 signal generated by MNP$\mathrm{Mn}(\mathrm{II})$-labeled BMSCs was observed in the right thigh after injection, and was maintained for at least 28 days; no hyperintense T1 signal was observed in the right thigh before injection. As shown in Figure 6, on day 1 after injection, the relative SI of the hyperintense T1 signal region was the highest at all time points, and the relative SI decreased gradually over time. On day 28 after injection, the SI was maintained at a relatively stable level, sufficient to allow for monitoring of engrafted cells. In addition, the area of the hyperintense T1 signal increased gradually within 4 days after injection. After reaching a maximum on day 4 after injection, the area decreased gradually over time. These results supported the suitability of MNP-Mn(II) as novel cell MR tracers for use in the long-term monitoring of the fate of engrafted BMSCs in vivo.

Compared with other positive MR cell tracers, such as gadolinium-based MR contrast agents, the main advantage of the novel MNP-Mn(II) is their high level of biosafety; the main components (melanin and manganese) are endogenous substances found in the human body. Hence, MNP-Mn(II) have almost no cytotoxicity in labeled BMSCs, even when used at a very high concentration. Additionally, MNP-Mn(II) can be easily taken up and internalized by BMSCs only by co-incubation without any transfection agents. In addition, BMSCs labeled with MNP-Mn(II) can generate a brighter 

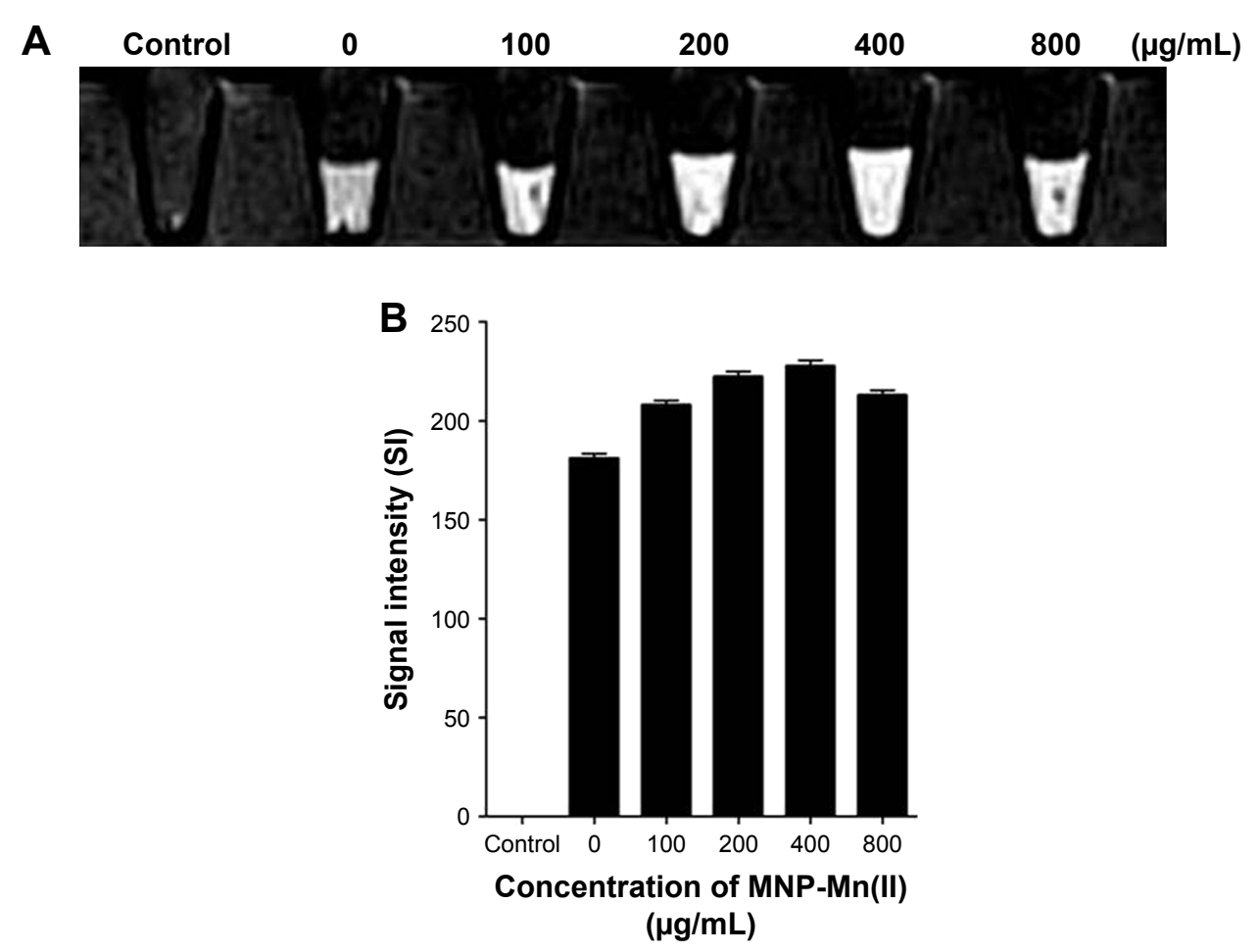

Figure 5 (A) TI-weighted images of BMSCs labeled with MNP-Mn(II) at various concentrations (0, 100, 200, 400, and $800 \mu \mathrm{g} / \mathrm{mL})$ in vitro. PBS was used as the control group. (B) MRI signal intensity of BMSCs labeled with MNP-Mn(II) at various concentrations (0, 100, 200, 400, and $800 \mu \mathrm{g} / \mathrm{mL}$ ).

Abbreviations: BMSCs, bone marrow-derived stem cells; MNP-Mn(II), manganese (II) ions chelated to melanin nanoparticles; MRI, magnetic resonance imaging; PBS, phosphate-buffered saline.

signal in T1-weighted images both in vitro and in vivo, owing to the higher T1 relaxivity of MNP-Mn(II), and the brighter signal can also be maintained for a long duration.

\section{Conclusion}

In summary, our findings demonstrated that MNP-Mn(II) had potential applications as MR stem cell tracers in vivo, owing to their excellent properties. We believe that these novel nanoparticles will be useful in studies of engrafted stem cells. In future studies, we will aim to further improve the nanostructures of the novel nanoparticles to achieve stem cell-based multimodal imaging and treatment integration, by incorporating other functional groups, such as ${ }^{64} \mathrm{Cu}^{2+}, \mathrm{Fe}^{3+}$, and sorafenib, into the nanoparticles. ${ }^{23,24}$

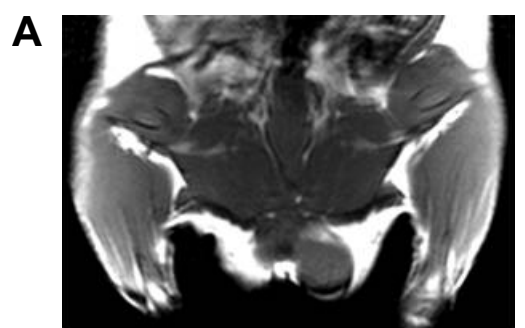

Not engrafted

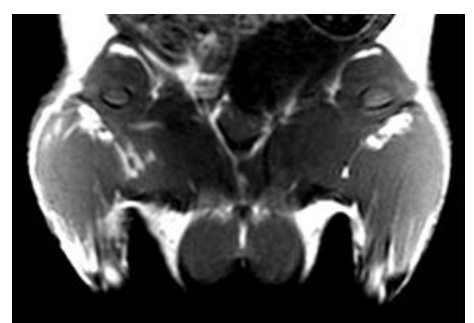

T1WI engrafted, day 4

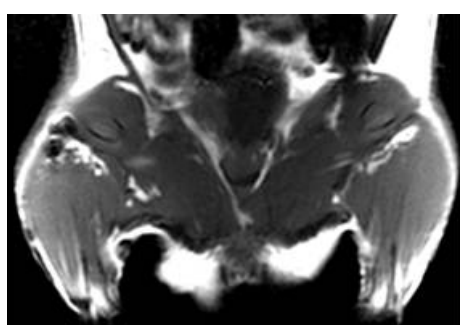

T1WI engrafted, day 1

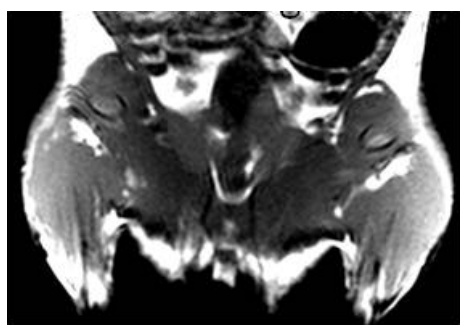

T1WI engrafted, day 7

Figure 6 (Continued) 


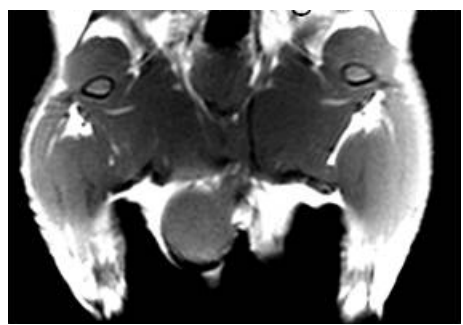

T1WI engrafted, day 10

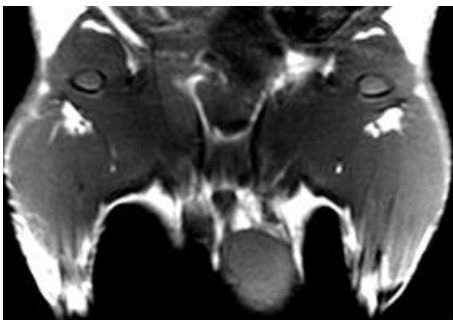

T1WI engrafted, day 21

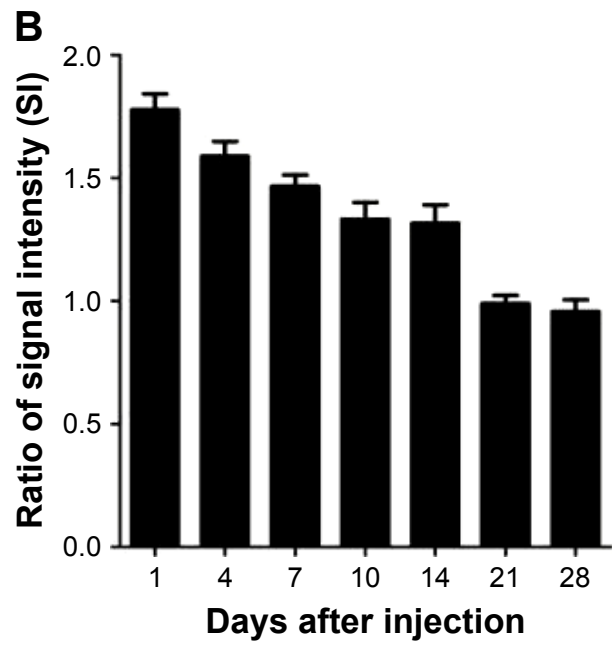

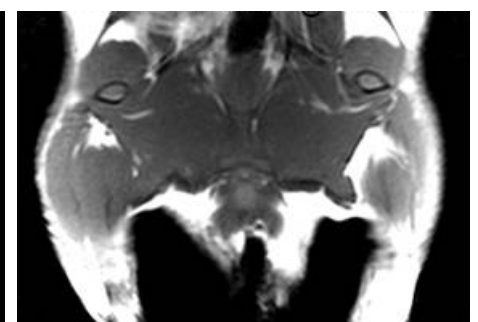

T1WI engrafted, day 14

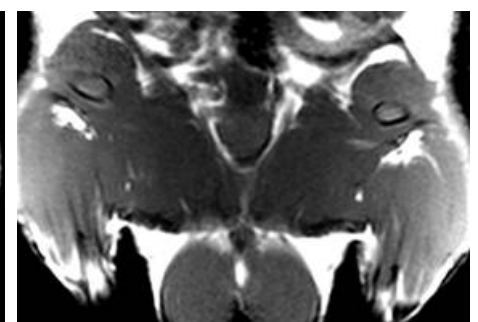

T1WI engrafted, day 28

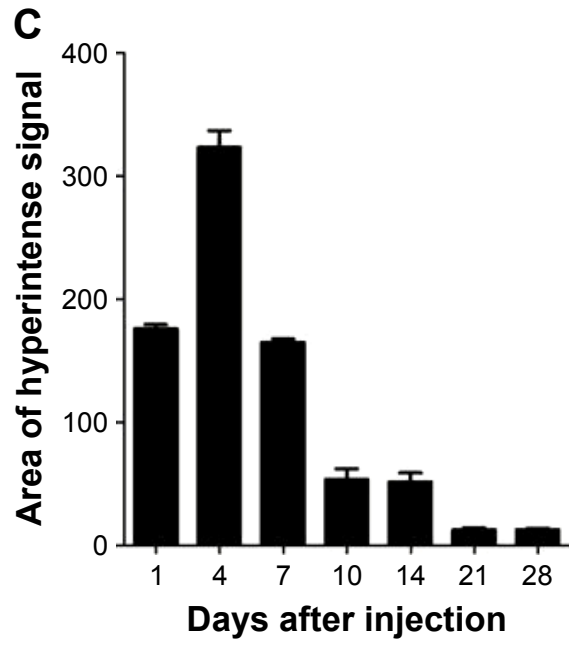

Figure 6 (A) TIWls of rats before MNP-Mn(II)-labeled BMSCs were injected into the right hip. TIWls of rats after MNP-Mn(II)-labeled BMSCs were injected into the right hip on days I, 4, 7, I4, 2I, and 28. Hyperintense TI signals in the right hip represented the presence of engrafted MNP-Mn(II)-labeled BMSCs in vivo. (B) The relative signal intensity of the MNP-Mn(II)-labeled BMSC injection region and the adjacent soft tissue over time. (C) The area of time-dependent hyperintense signals generated by MNP-Mn(II)-labeled BMSCs.

Abbreviations: BMSCs, bone marrow-derived stem cells; MNP-Mn(II), manganese (II) ions chelated to melanin nanoparticles; MRI, magnetic resonance imaging; PBS, phosphate-buffered saline; WI, weighted image.

\section{Acknowledgments}

This work was financially supported by the National Natural Science Foundation of China (grant numbers 81371628, 81571747, and 81771907), Overseas Students Science and Technology Projects, Shanxi Scholarship Council of China (number 2015057), Key Research and Development Project of Shanxi Province (numbers 2016021 and 201703D321015-3), Science and Technology Innovation Team Project (number 201705D131026), Scientific and Technological Achievements Transformation Project of Shanxi Province (number 201704D131006), and Shanxi Province Science Foundation for Youths (number 201701D221257).

\section{Disclosure}

The authors report no conflicts of interest in this work.

\section{References}

1. Neuss S, Schneider RK, Tietze L, Knuchel R, Jahnen-Dechent W. Secretion of fibrinolytic enzymes facilitates human mesenchymal stem cell invasion into fibrin clots. Cells Tissues Organs. 2010;191(1) 36-46.

2. Talavera-Adame D, Wu G, He Y, Ng TT, Gupta A, Kurtovic S, et al. Endothelial cells in co-culture enhance embryonic stem cell differentiation to pancreatic progenitors and insulin-producing cells through BMP signaling. Stem Cell Rev. 2011;7(3):532-543

3. Maijenburg MW, Gilissen C, Melief SM, Kleijer M, Weijer K, Ten Brinke A, et al. Nuclear receptors Nur77 and Nurr1 modulate mesenchymal stromal cell migration. Stem Cells Dev. 2012;21(2):228-238.

4. Barry FP, Murphy JM. Mesenchymal stem cells: clinical applications and biological characterization. Int J Biochem Cell Biol. 2004;36(4): $568-584$. 
5. Shen Y, Shao Y, He H, Tan Y, Tian X, Xie F, et al. Gadolinium(3+)doped mesoporous silica nanoparticles as a potential magnetic resonance tracer for monitoring the migration of stem cells in vivo. Int $J$ Nanomedicine. 2013;8:119-127.

6. Cen P, Chen J, Hu C, Fan L, Wang J, Li L. Noninvasive in-vivo tracing and imaging of transplanted stem cells for liver regeneration. Stem Cell Res Ther. 2016;7(1):143.

7. Shichinohe H, Kuroda S, Lee JB, Nishimura G, Yano S, Seki T, et al. In vivo tracking of bone marrow stromal cells transplanted into mice cerebral infarct by fluorescence optical imaging. Brain Res Brain Res Protoc. 2004;13(3):166-175.

8. Tseng CL, Shih IL, Stobinski L, Lin FH. Gadolinium hexanedione nanoparticles for stem cell labeling and tracking via magnetic resonance imaging. Biomaterials. 2010;31(20):5427-5435.

9. Kim KS, Park W, Na K. Gadolinium-chelate nanoparticle entrapped human mesenchymal stem cell via photochemical internalization for cancer diagnosis. Biomaterials. 2015;36:90-97.

10. Wang J, Xie J, Zhou X, Cheng Z, Gu N, Teng G, et al. Ferritin enhances SPIO tracking of C6 rat glioma cells by MRI. Mol Imaging Biol. 2011; 13(1):87-93.

11. Jokerst JV, Khademi C, Gambhir SS. Intracellular aggregation of multimodal silica nanoparticles for ultrasound-guided stem cell implantation. Sci Transl Med. 2013;5(177):177ra35.

12. Kim T, Momin E, Choi J, Yuan K, Zaidi H, Kim J, et al. Mesoporous silica-coated hollow manganese oxide nanoparticles as positive T1 contrast agents for labeling and MRI tracking of adipose-derived mesenchymal stem cells. J Am Chem Soc. 2011;133(9):2955-2961.

13. Gao J, Chen K, Xie R, Xie J, Lee S, Cheng Z, et al. Ultrasmall nearinfrared non-cadmium quantum dots for in vivo tumor imaging. Small. 2010;6(2):256-261.

14. Hu X, Zhu J, Li X, Zhang X, Meng Q, Yuan L, et al. Dextran-coated fluorapatite crystals doped with $\mathrm{Yb} 3+/ \mathrm{Ho} 3+$ for labeling and tracking chondrogenic differentiation of bone marrow mesenchymal stem cells in vitro and in vivo. Biomaterials. 2015;52:441-451.

15. Cheng L, Wang C, Ma X, Wang Q, Cheng Y, Wang H, et al. Multifunctional upconversion nanoparticles for dual-modal imaging-guided stem cell therapy under remote magnetic control. Adv Funct Mater. 2013;23(3):272-280.

16. Na HB, Lee JH, An K, Park YI, Park M, Lee IS, et al. Development of a $\mathrm{T} 1$ contrast agent for magnetic resonance imaging using $\mathrm{MnO}$ nanoparticles. Angew Chem Int Ed Engl. 2007;46(28):5397-5401.
17. Tran LA, Krishnamurthy R, Muthupillai R, Cabreira-Hansen Mda G, Willerson JT, Perin EC, et al. Gadonanotubes as magnetic nanolabels for stem cell detection. Biomaterials. 2010;31(36):9482-9491.

18. Geng K, Yang ZX, Huang D, Yi M, Jia Y, Yan G, et al. Tracking of mesenchymal stem cells labeled with gadolinium diethylenetriamine pentaacetic acid by $7 \mathrm{~T}$ magnetic resonance imaging in a model of cerebral ischemia. Mol Med Rep. 2015;11(2):954-960.

19. Lee JH, Koretsky AP. Manganese enhanced magnetic resonance imaging. Curr Pharm Biotechnol. 2004;5(6):529-537.

20. Tan M, Ye Z, Jeong EK, Wu X, Parker DL, Lu ZR. Synthesis and evaluation of nanoglobular macrocyclic Mn(II) chelate conjugates as non-gadolinium(III) MRI contrast agents. Bioconj Chem. 2011;22(5): 931-937.

21. Riley PA. Melanin. Int J Biochem Cell Biol. 1997;29(11):1235-1239.

22. Dong H, Liu Z, Zhong H, Yang H, Zhou Y, Hou Y, et al. Melaninassociated synthesis of SERS-active nanostructures and the application for monitoring of intracellular melanogenesis. Nanomaterials (Basel). 2017;7(3):70.

23. Zhang R, Fan Q, Yang M, Cheng K, Lu X, Zhang L, et al. Engineering melanin nanoparticles as an efficient drug-delivery system for imagingguided chemotherapy. Adv Mater. 2015;27(34):5063-5069.

24. Fan Q, Cheng K, Hu X, Ma X, Zhang R, Yang M, et al. Transferring biomarker into molecular probe: melanin nanoparticle as a naturally active platform for multimodality imaging. J Am Chem Soc. 2014;136(43): 15185-15194.

25. National Research Council. Guide for the care and use of laboratory animals, 8th ed. Washington, DC: National Academies Press; 2011.

26. Zhang RP, Wang LJ, He S, Xie J, Li JD. Effects of magnetically guided, SPIO-labeled, and neurotrophin-3 gene-modified bone mesenchymal stem cells in a rat model of spinal cord injury. Stem Cells Int. 2016; 2016:2018474.

27. Wang J, Jokerst JV. Stem cell imaging: tools to improve cell delivery and viability. Stem Cells Int. 2016;2016:9240652.

28. Reimer P, Parizel PM, Meaney JFM, Stichnoth FA, editors. Clinical MR imaging: A practical approach, 3rd ed. Secaucus, NJ: Springer; 2010.

29. Crich SG, Biancone L, Cantaluppi V, Duò D, Esposito G, Russo S, et al. Improved route for the visualization of stem cells labeled with a Gd-/ Eu-chelate as dual (MRI and fluorescence) agent. Magn Reson Med. 2004;51(5):938-944. 


\section{Supplementary materials Synthesis of water-dispersible melanin nanoparticles (MNPs)}

Water-dispersible MNPs were synthesized according to a previously described procedure. ${ }^{1}$ Briefly, $20 \mathrm{mg}$ melanin (Sigma-Aldrich, St Louis, MO, USA) was dissolved in $4 \mathrm{~mL}$ of $0.1 \mathrm{M} \mathrm{NaOH}$ (Sigma-Aldrich) using an ultrasonic bath. $\mathrm{HCl}$ (37 wt \%; Sigma-Aldrich) was dripped gradually to adjust the $\mathrm{pH}$ to $7.0-8.0$, and an ultrasonic processor was used to decentralize MNPs. The obtained MNPs aqueous solution was transferred to an ultrafiltration centrifuge tube (Millipore Amicon Ultra, $30 \mathrm{kDa}$, EMD Millipore, Billerica, MA, USA) and then purified by centrifugation $(4,000-5,000 \times g, 30 \mathrm{~min})$ and washed with deionized water several times until the filtered water became clear. About $1 \mathrm{~mL}$ of MNP aqueous solution was left in the tube and then lyophilized for $24 \mathrm{~h}$ using a vacuum freeze dryer. Ultimately, $14 \mathrm{mg}$ of black solid water-dispersible MNPs was obtained.

\section{Surface modification of MNPs}

The surface of MNPs was further modified by polyethylene glycol (PEG, $\mathrm{NH}_{2}$-PEG5000- $\mathrm{NH}_{2}, 5 \mathrm{kDa}$; Laysan Bio, Arab, AL, USA). The previously lyophilized MNPs were redissolved in alkaline solution, and the $\mathrm{pH}$ was adjusted to 9.5 by adding $0.1 \mathrm{M} \mathrm{NaOH}$ aqueous solution dropwise. Approximately $70 \mathrm{mg}$ PEG was dissolved in $\sim 8 \mathrm{~mL}$ ultrapure water in an ultrasonic bath, and the $\mathrm{pH}$ was also adjusted to 9.5 by adding $0.1 \mathrm{M} \mathrm{NaOH}$ aqueous solution dropwise. Under the protection of nitrogen, the PEG and MNP aqueous solutions were mixed together and stirred for $24 \mathrm{~h}$ at room temperature using a magnetic stirrer. The solution was then transferred to an ultrafiltration centrifuge tube and washed five times with deionized water by centrifugation $(4,000-5,000 \times g, 15 \mathrm{~min})$ to remove the free PEG. The $\mathrm{pH}$ of the final PEG-MNP aqueous solution was nearly 7.0.

\section{Synthesis of $\mathrm{Mn}^{2+}$-PEG-MNPs}

In total, $4 \mathrm{~mL}$ of $2.5 \mathrm{mg} / \mathrm{mL}$ MNP aqueous solution was mixed with $100 \mu \mathrm{L}$ of $100 \mathrm{mg} / \mathrm{mL} \mathrm{MnCl}_{2}$ aqueous solution and then stirred gently at $40^{\circ} \mathrm{C}$ for $2 \mathrm{~h}$. To ensure that the MNPs were fully chelated with $\mathrm{Mn}^{2+}$ ions, moderately excessive $\mathrm{Mn}^{2+}$ ions were required. The molar ratio of MNPs and $\mathrm{Mn}^{2+}$ ions in the resulting chelate was $\sim 1: 200$. The obtained chelation products were then purified using a PD-10 column
(GE Healthcare, Chicago, IL, USA) to removed free $\mathrm{Mn}^{2+}$ and $\mathrm{Cl}^{-}$.

\section{Characterization of $\mathrm{Mn}^{2+}$-PEG-MNPs}

The morphology of $\mathrm{Mn}^{2+}$-PEG-MNPs was observed using a transmission electron microscope (TEM; JEM-1011; JEOL, Tokyo, Japan). The hydrodynamic diameters were measured by dynamic light scattering (DLS; Zetasizer Nano ZS90; Malvern Instruments, UK). TEM images (Figure S1B and C) and sizes distribution of DLS (Figure S1D) showed monodispersed spherical nanoparticles of $\sim 5 \mathrm{~nm}$ (approximate range $=3-7 \mathrm{~nm}$ ).

The $\mathrm{Mn}^{2+}$ ion concentration in $10 \mathrm{~mL}$ of $0.02 \mathrm{mg} / \mathrm{mL}$ $\mathrm{Mn}^{2+}$-PEG-MNP aqueous solution was measured by inductively coupled plasma mass spectrometry (ICP-MS). The ICP-MS results revealed that the $\mathrm{Mn}^{2+}$ ion concentration was about $1.74 \mathrm{mg} / \mathrm{L}$, and the quantity of $\mathrm{Mn}^{2+}$ ions chelated to a single MNP was about 69.5.

The stability of $\mathrm{Mn}^{2+}$ ion-chelated MNPs was also analyzed in phosphate-buffered saline (PBS; $\mathrm{pH}=7.4$ ). As shown in Figure S1E, only about $3 \% \mathrm{Mn}^{2+}$ ions were released from $\mathrm{Mn}^{2+}$-PEG-MNPs, and the quantity of released $\mathrm{Mn}^{2+}$ ions did not increase further during subsequent incubation times, even at $48 \mathrm{~h}$. These results indicated that $\mathrm{Mn}^{2+}-\mathrm{PEG}-$ MNPs had high stability.

\section{Fontana-Masson staining}

Fontana-Masson staining (Solarbio, G2032) was performed to identify the MNP-Mn ${ }^{2+}$ labeling rate of bone marrowderived stem cells (BMSCs). After labeling with MNP- $\mathrm{Mn}^{2+}$, the BMSCs were fixed with paraformaldehyde for $15 \mathrm{~min}$ at room temperature, washed three times with PBS, and then incubated in ammoniacal silver solution for $35 \mathrm{~min}$ at $56^{\circ} \mathrm{C}$ in a darkroom. Next, samples were rinsed five times with deionized water, soaked in 5\% sodium thiosulfate solution for $5 \mathrm{~min}$ at room temperature, and then rinsed again with running pure water for 3 min. Finally, samples were counterstained with neutral red staining solution for $5 \mathrm{~min}$ at room temperature in a darkroom. The labeled BMSCs were observed using a phase-contrast microscope (E100; Nikon, Tokyo, Japan).

Black particles representing $\mathrm{Mn}^{2+}$-PEG-MNPs were observed in the cytoplasm of labeled BMSCs, while no black particles were observed in the cytoplasm of unlabeled BMSCs. Additionally, the $\mathrm{Mn}^{2+}$-PEG-MNP labeling rate of the BMSCs was nearly $100 \%$ (Figure S2). 

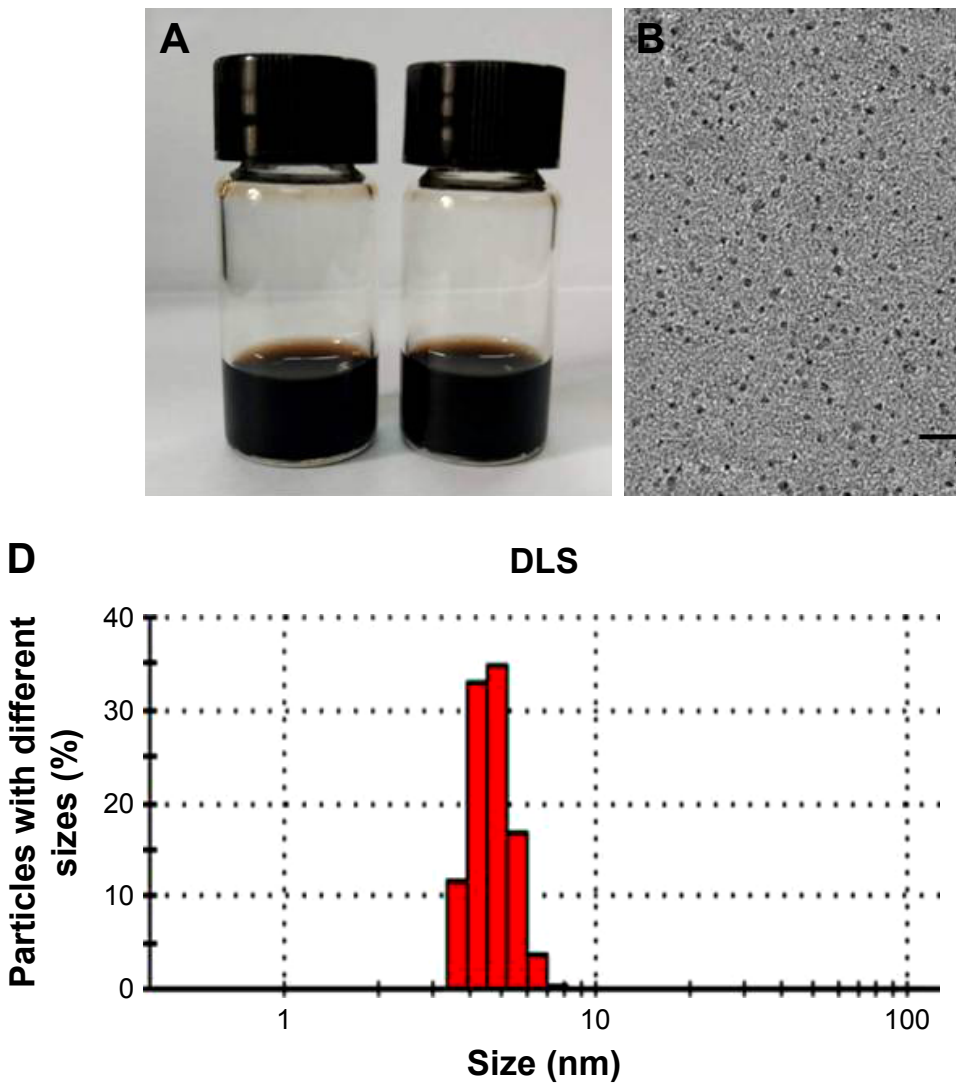
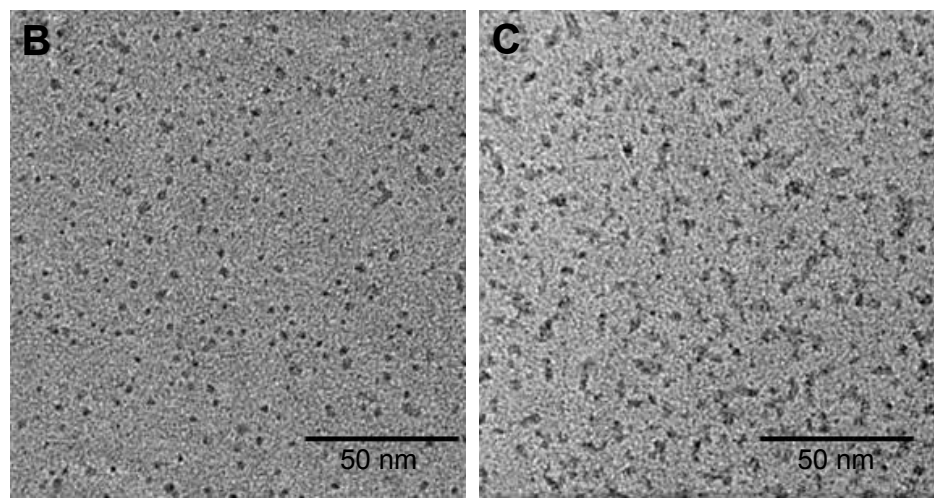

$\mathbf{E}$

Stability assay

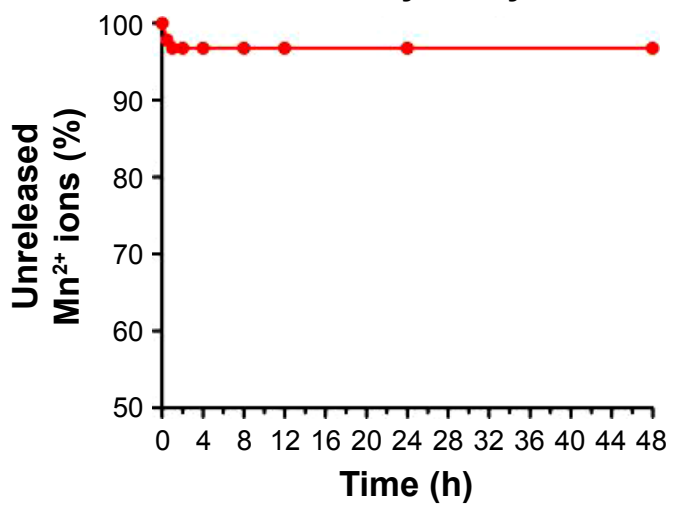

Figure SI Characterization of the physical properties of Mn²+-PEG-MNPs. (A) From left to right: $2 \mathrm{mg} / \mathrm{mL}$ PEG-MNPs and $2 \mathrm{mg} / \mathrm{mL}$ Mn ${ }^{2+}-$ PEG-MNPs. (B) TEM image of $\mathrm{Mn}^{2+}$-PEG-MNPs (scale bar $=50 \mathrm{~nm}$ ). (C) TEM image of MNPs (scale bar $=50 \mathrm{~nm}$ ). (D) Particle-size distribution of Mn ${ }^{2+}-\mathrm{PEG}-\mathrm{MNPs}$. (E) Stability analysis of Mn ${ }^{2+}-\mathrm{PEG}-\mathrm{MNPs}$ in PBS ( $\mathrm{pH}=7.4)$.

Abbreviations: DLS, dynamic light scattering; h, hours; MNPs, melanin nanoparticles; PBS, phosphate-buffered saline; PEG, polyethylene glycol; TEM, transmission electron microscope.
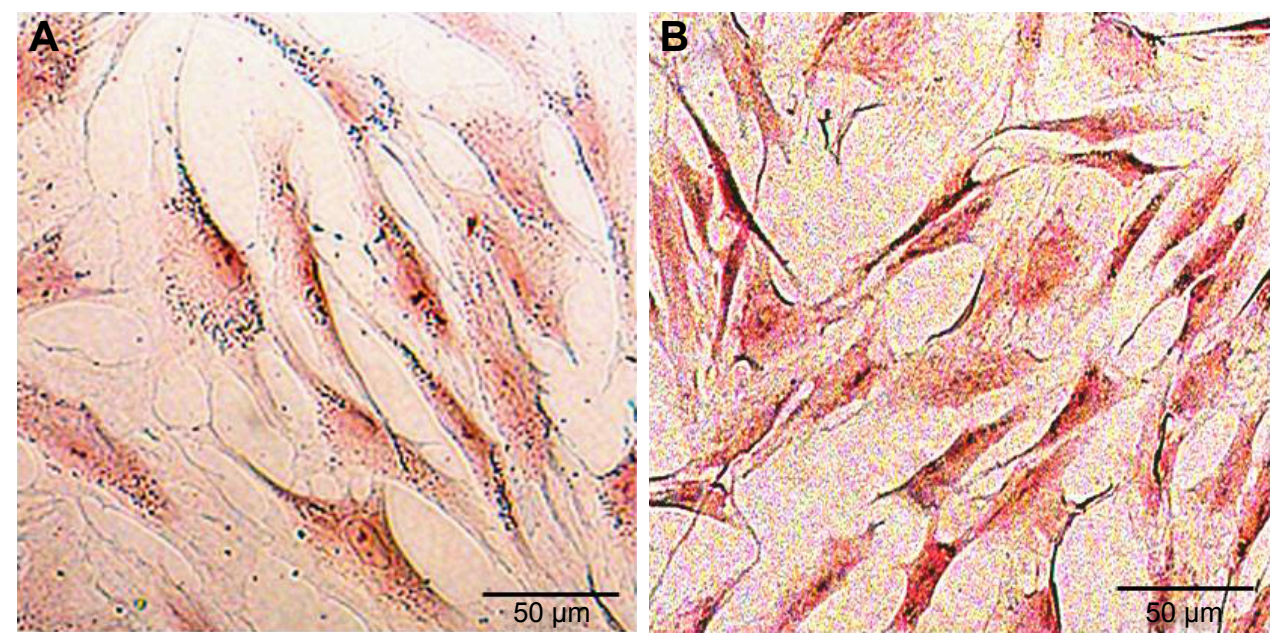

Figure S2 Fontana-Masson staining of Mn ${ }^{2+}$-PEG-MNP-labeled and unlabeled BMSCs. (A) Black particles representing Mn ${ }^{2+}-$ PEG-MNPs were observed in the cytoplasm of labeled BMSCs. (B) Black particles were not observed in the cytoplasm of labeled BMSCs. Magnification $=200 \times$. Scale bars $=50 \mu \mathrm{m}$.

Abbreviations: BMSCs, bone marrow-derived stem cells; MNPs, melanin nanoparticles; PEG, polyethylene glycol.

\section{Reference}

1. Fan Q, Cheng K, Hu X, Ma X, Zhang R, Yang M, et al. Transferring biomarker into molecular probe: melanin nanoparticle as a naturally active platform for multimodality imaging. J Am Chem Soc. 2014;136(43): 15185-15194. 
International Journal of Nanomedicine

Dovepress

\section{Publish your work in this journal}

The International Journal of Nanomedicine is an international, peerreviewed journal focusing on the application of nanotechnology in diagnostics, therapeutics, and drug delivery systems throughou the biomedical field. This journal is indexed on PubMed Central, MedLine, CAS, SciSearch ${ }^{\circledR}$, Current Contents ${ }^{\circledR} /$ Clinical Medicine,
Journal Citation Reports/Science Edition, EMBase, Scopus and the Elsevier Bibliographic databases. The manuscript management system is completely online and includes a very quick and fair peer-review system, which is all easy to use. Visit http://www.dovepress.com/ testimonials.php to read real quotes from published authors.

Submit your manuscript here: http://www.dovepress.com/international-journal-of-nanomedicine-journal 